\title{
Evaluación del sistema de marcaje físico visible (V.I.E) en cachama blanca (Piaractus brachypomus)
}

\author{
Evaluating a visible implant elastomer (VIE) tag system for \\ cachama/pacu (Piaractus brachypomus)
}

\section{Avaliação do sistema de marcação física visível (VIE) em cachama blanca (Piaractus brachypomus)}

\author{
Gerald F. Bernal-Buitrago ${ }^{1 *}$, Jeferson A. Valderrama-Diaz ${ }^{*}$, Jhon M Santos-Salamanca ${ }^{*}$; \\ Ana Milena Riaño-Gómez; Pablo E Cruz-Casallas ${ }^{5 *}$; Víctor M. Medina-Robles ${ }^{6 *}$ \\ 1 Zoot, Estudiante de Maestría en Acuicultura, Instituto de Acuicultura de la Universidad de los Llanos - IALL, Villavicencio, \\ Meta - Colombia; \\ 2 Universidad de la Salle, Bogotá - Colombia; Zoot; \\ 3 Estudiante de Medicina Veterinaria y Zootecnia, Universidad de los Llanos, Villavicencio, Meta - Colombia; \\ 5 MVZ, MSc, PhD; \\ 6 MVZ, MSc, Profesor Facultad de Ciencias Agropecuarias y Recursos Naturales, Instituto de Acuicultura de los Llanos - \\ IALL, Villavicencio, Meta - Colombia. \\ * Grupo de Investigación sobre Reproducción y Toxicología de Organismos Acuáticos - GRITOX, Instituto de Acuicultura - \\ IALL, Facultad de Ciencias Agropecuarias y Recursos Naturales, Universidad de los Llanos, Villavicencio, Meta-Colombia. \\ Email:vmmedinarobles@unillanos.edu.co
}

Recibido: 26 agosto de 2015

Aceptado: 18 agosto de 2016

\begin{abstract}
Resumen
La identificación de individuos en la piscicultura se ha venido estableciendo como un proceso estándar, lo que implica la utilización de un sistema de marcaje eficiente, práctico y económico. Este estudio evaluó el sistema de marcaje físico visible (V.I.E) con relación al crecimiento, sobrevivencia, retención y visibilidad en alevinos de cachama blanca (Piaractus brachypomus). Para este propósito, fueron usados 400 peces en dos experimentos con 200 peces cada uno; experimento $1(4.5 \pm 0.9 \mathrm{~g})$ y experimento $2(8.26 \pm 1.94 \mathrm{~g})$ evaluadas durante un periodo de 30 días, repartidos al azar en 4 tanques de 250 litros. Después de un periodo de aclimatación de 8 días y siguiendo las indicaciones del fabricante, se marcaron 128 peces con 8 colores: Azul, rojo, verde, amarillo, naranja, rosado (fluorescentes), café y morado (no fluorescentes), en dos posiciones corporales (dorso y opérculo) definidas en ensayos preliminares. Los animales no marcados (72) pero sometidos al mismo procedimiento, se utilizaron como control. Los peces en su totalidad, se midieron y pesaron al inicio y al final de cada experimento. Para ninguno de los experimentos se determinó efecto del implante sobre el crecimiento $(p<0,05)$ entre los peces marcados y el control. La sobrevivencia en ambos experimentos fue del $100 \%$. La retención en el dorso y opérculo en promedio para los dos experimentos fue de $94.11 \%$ y $57.52 \%$, respectivamente. La visibilidad con luz UV y sin luz UV en el opérculo fue de $96.6 \%$ y $77.7 \%$ y para el dorso fue de $92.6 \%$ y $61.5 \%$, respectivamente. En conclusión la posición más viable para el uso del V.I.E en animales de baja talla, es el dorso debido a su alto porcentaje de retención, preferiblemente utilizando los colores fluorescentes bajo exposición a luz UV.
\end{abstract}

Palabras clave: crecimiento, fluorescencia, identificación, implante. 


\begin{abstract}
Identifying individuals in fish-farming has become standard procedure, thereby implying the use of an efficient, practical and economic tagging system. This study evaluated the visible implant elastomer (VIE) system related to growth, survival, retention and visibility in cachama/pacu (Piaractus brachypomus) fry. The study involved using 400 fish in two experiments (200 fish in each experiment): experiments $1(4.5 \pm 0.9 \mathrm{~g})$ and $2(8.26 \pm 1.94 \mathrm{~g})$ were evaluated over a 30-day period, the fry being randomly distributed in four 250-liter tanks. Following an 8-day acclimatisation period, 128 fish were tagged with 8 colours (following the manufacturer's instructions): blue, red, green, yellow, orange, pink (fluorescent), Brown and purple (non-fluorescent); they were tagged on two parts of the body (dorsal/back and operculum/cheek area) which had already been defined in preliminary trials. Untagged fry (72) kept in the same conditions were used as control. All fry were measured and weighed at the start and finish of each experiment. It was determined that the implant/tag had no effect on growth $(p<0.05)$ in either tagged fry or control. The survival of both types of fry in both experiments was $100 \%$. Tag retention in both experiments regarding the back was $94.11 \%$ on average and $57.52 \%$ for the cheek area. Visibility with UV light was $96.6 \%$ on the cheek area and $92.6 \%$ for the back and without UV light $77.7 \%$ for the cheek area and $61.5 \%$ for the back. It can thus be stated that the dorsal/back area is the most viable position for using VIE with low-weight fry due to the high retention percentage, preferably using fluorescent reporters under UV light.
\end{abstract}

Key words: growth, fluorescence, identification, implant.

\title{
Resumo
}

A identificação de indivíduos na piscicultura se tem estabelecido como um processo regular, o que implica a utilização de um sistema de marcação eficiente, prático e económico. Este estudo avaliou o sistema de marcação física visível em relação ao crescimento, sobrevivencia, retenção e visibilidade em alevinos de cachama blanca (Piaractus brachypomus). Para este propósito, 400 peixes foram usados em dois experimentos com 200 peixes cada, experimento 1 (4.5 $\pm 0.9 \mathrm{~g}$ ) e experimento $2(8.26 \pm 1.94 \mathrm{~g})$ avaliados durante um período de 30 dias, repartidos ao acaso em quatro tanques de 250 litros. Uma vez aclimatados os peixes e seguindo as indicações do fabricante, marcaram-se 128 peixes com oito cores: Azul, vermelho, verde, amarelo, laranja, rosado (fluorescentes), café e morado (não fluorescentes), em duas posições corporais (Dorso e opérculo) definidas em ensaios preliminares. Os animais não marcados (72) submetidos ao mesmo procedimento se utilizaram como controle. Os peixes em sua totalidade foram medidos e pesados ao início e ao final da cada experimento. Não foi determinado efeito do implante sobre o crescimento $(p<0,05)$ entre os peixes marcados e o controle para nenhum dos experimentos. A sobrevivência em ambos experimentos foi de $100 \%$. A retenção no dorso e opérculo em média foi de $94.11 \%$ e $57.52 \%$, respectivamente para os dois experimentos. A visibilidade com luz UV e sem luz UV no opérculo foi de $96.6 \%$ e $77.7 \%$ e para o dorso foi de $92.6 \%$ e $61.5 \%$, respectivamente. Em conclusão a posição mais viável para o uso do V.I.E em animais de baixa talha, é o dorso devido a sua alta percentagem de retenção, preferivelmente utilizando as cores fluorescentes baixo exposição a luz UV.

Palavras-chave: crescimento, fluorescencia, identificação, implante.

\section{Introducción}

La piscicultura según la FAO (2014), continúa siendo la esfera de producción de alimentos de origen animal de crecimiento más rápido y actualmente produce cerca de la mitad del suministro total de pescado comestible, con lo cual realiza una contribución fundamental a la mitigación de la pobreza, siendo la alternativa más viable para cubrir la demanda y subsanar la seguridad alimentaria en los próximos años, a través del aumento en la eficiencia productiva, lo que implica mejorar las condiciones de manejo, infraestructura y calidad genética de las especies.

En este contexto el reto de la cadena piscícola colombiana es involucrar los pilares fundamentales de una manera más global e integral, donde se le dé mayor importancia al mejoramiento genético de las especies nativas con el propósito de transformar al sector en uno tecnificado y líder de la economía nacional, capaz de trascender al mercado internacional (Leiva et al., 2007).

La cachama blanca (Piaractus brachypomus) de acuerdo con Sastre et al., (2009) es considerada la especie nativa de más alto potencial piscícola, debido a su resistencia a enfermedades, hábitos omnívoros y rusticidad al manejo en cautiverio. Sin embargo, según Nascimento et al., (2010) la especie no ha podido avanzar significativamente en términos de tasas de crecimiento, conversión alimenticia, consolidación de su comercialización y consumo, y a su vez aspectos relacionados con la creciente necesidad de dietas más nutricionales por parte de la población humana, en este sentido, un desarrollo sostenible del cultivo de la especie en Colombia, además de optimizar los sistemas de producción, reproducción y cruces está ceñido al conocimiento genético de la especie (MesaGranda et al., 2006, Mesa-Granda y Botero-Aguirre, 
2007). Sin embargo, la implantación de un programa genético requiere de información familiar a través de la eficiente identificación de los grupos familiares y de los peces individualmente; con respecto a Bernal y Gallego (2016), lo cual actualmente está establecido como la industria estándar para especies acuícolas, que a su vez conlleva a implementar un sistema de identificación eficaz en animales de baja talla, confiable y de bajo costo .

Uno de los sistemas de marcaje más utilizado en la industria acuícola por tener la ventaja de ser rápido, de bajo costo y fácil de usar en peces de tamaño pequeño, es el sistema de marcaje interno subcutáneo Visible Implant Elastomer (V.I.E; North-west Marine Technology Inc.), está inclusive por encima del Passive Integrated Transponder (PIT; EiD Ibérica SA - TROVAN) que por ser electrónico de aplicación interna se ha evaluado ampliamente aunque con la desventaja de ser costoso y limitante su uso en animales de tamaño pequeño.

El VIE es un marcador que a pesar de que ha sido usado con éxito en distintas especies de peces (Bailey et al., 1998, Baras et al., 1999, Baras et al., 2000, Astorga et al., 2005, Brennan et al., 2007), requiere una evaluación específica para cada una de ellas, debido a que su utilidad viene determinada por sus diferentes grados de aceptación, ya sea por factores como la talla, el color de la piel o la capacidad de soportar un manejo intenso. Es por esto que el objetivo de este trabajo consistió en evaluar el sistema de marcaje físico visible VIE en relación con el crecimiento, sobrevivencia, retención y visibilidad en alevinos de cachama blanca.

\section{Materiales y métodos}

\section{Comité de ética}

Todos los procedimientos fueron realizados de conformidad con las normas y procedimientos para el uso de animales de laboratorio, descritas por el Comité on Care and Use of Laboratory Animal Resources - National Research Counsil, USA (1996). De igual forma, los procedimientos del presente estudio, contaron con la aprobación del Comité de Bioética de la Universidad de los Llanos.

\section{Localización}

El trabajo fue llevado a cabo en las instalaciones del Grupo de Investigación sobre Reproducción y Toxicología de Organismos Acuáticos GRITOX del Instituto de Acuicultura de la Universidad de los Llanos (IALL-UNIL-
LANOS), ubicado en la ciudad de Villavicencio-Meta (Colombia), a una altura de $418 \mathrm{msnm}$, temperatura promedio de $26{ }^{\circ} \mathrm{C}$, humedad relativa del $75 \%$ y precipitación pluvial de $4.050 \mathrm{~mm}$, cuyas coordenadas son $4^{\circ} 04^{\prime} 24.8^{\prime \prime}$ de Latitud Norte y $73^{\circ} 34^{\prime} 56.2^{\prime \prime}$ de Latitud oeste a 12 kilómetros de la vía que conduce de la ciudad de Villavicencio al municipio de Puerto López.

\section{Material biológico}

Se utilizaron 400 alevinos de cachama blanca clínicamente sanos, divididos en dos grupos, uno de ellos con un peso corporal promedio de $4,5 \pm 0.9 \mathrm{~g}$ (experimento 1) y el segundo con $8.38 \pm 1.98 \mathrm{~g}$ (experimento $2)$, provenientes de un mismo desove realizado en la estación piscícola Agua Verde, ubicada en el municipio de Cumaral, Departamento del Meta.

\section{Calidad del agua}

Diariamente fue evaluada la temperatura $\left({ }^{\circ} \mathrm{C}\right)$ por medio de un termómetro digital MARTINI $\AA$ instruments EC59. Semanalmente se evaluaron las características físicas y químicas del agua, como $\mathrm{pH}$, oxígeno disuelto, amonio, dureza y alcalinidad ( $\mathrm{mg} / \mathrm{l})$ por medio de un kit comercial (Kit Hach FF1-A® - Hach Company) en cada uno de los tanques. Se realizaron recambios de agua del $30 \%$ de la columna del agua durante todo el estudio con una frecuencia de tres veces por semana. Antes de llevar a cabo el estudio, se desinfectaron todos los materiales como tanques, tapas, tuberías, piedras difusoras y demás tipo de elementos que tendrían contacto con los animales.

\section{Diseño experimental}

El experimento tuvo una duración de 30 días para cada uno de los experimentos. Los peces $(n=400)$ fueron dispuestos aleatoriamente en ocho tanques de polipropileno, con capacidad de 250 litros con dimensiones de $58 \times 118.5 \times 100 \mathrm{~cm}$, cuatro para cada experimento y 50 animales por tanque.

Pasados ocho días como periodo de adaptación y siguiendo las recomendaciones del fabricante (NorthWest Marine Technology Inc.), todos los peces fueron anestesiados con 2-fenoxietanol (300 ppm, JT Baker $\left.{ }^{\circledR}\right)$, para registrar el peso $(\mathrm{g})$, y la talla $(\mathrm{mm})$, posteriormente se marcaron animales aleatoriamente 128 peces con ocho colores: azul, rojo, verde, amarillo, naranja, rosado (fluorescentes), café y morado (no fluorescentes), en dos posiciones corporales (dorso y opérculo) definidas en ensayos preliminares (figura 1). Los animales no marcados (72) pero sometidos al mismo proce- 
dimiento se utilizaron como control. Adicionalmente se midió el tiempo (s) que duraba el proceso de marcaje. Los peces en su totalidad, se midieron y pesaron al inicio y al final de cada experimento.

La tasa de alimentación diaria durante el experimento fue del $12 \%$ de la biomasa repartida en tres tomas, con una dieta comercial de $45 \%$ de proteína cruda.

\section{Evaluación de la retención}

Con el fin de determinar los porcentajes de retención generados durante los 30 días en condiciones controladas de laboratorio, se definió que la perdida de una porción del implante no afectaba su evaluación, simplemente se observó su permanencia por lo que se definió como una variable cualitativa.

\section{Crecimiento}

La tasa de crecimiento específica se calculó mediante la expresión:

$$
\operatorname{SGR}(\%)=(\operatorname{Ln} P F-\operatorname{Ln} \mathrm{PI}) 100 / \Delta \mathrm{T}
$$

Donde $\mathrm{PF}, \mathrm{Pl}$ y $\Delta \mathrm{T}$ son peso final, peso inicial y periodo de estudio respectivamente.

\section{Visibilidad}

Se realizó un experimento adicional en donde se evaluaron dos grupos de animales, uno de ellos con animales de 30 días de marcados, vs animales de un día de implantados, se observó, cual o cuales de los ocho colores utilizados en la primera fase del proyecto es más visible (rosado, azul, rojo, verde, anaranjado, blanco, azul y morado) con y sin luz U.V en alevinos de cachama blanca. Para determinar la visibilidad del implante, se diseñó una encuesta basada en la medición

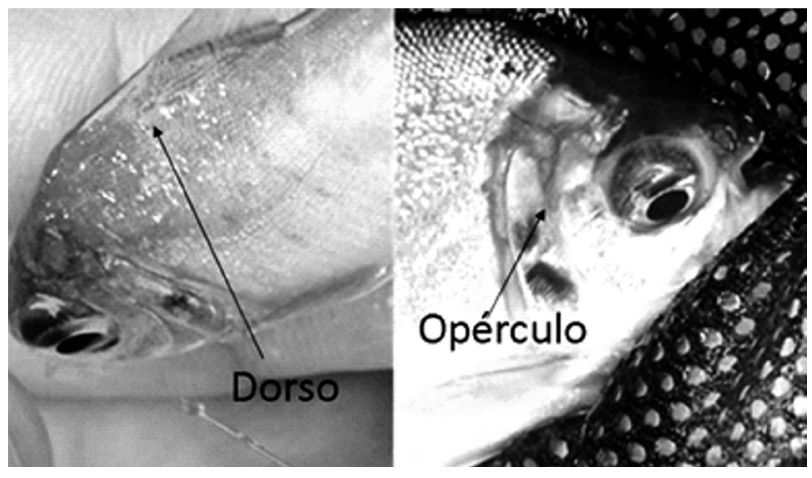

Figura 1. Diagrama de la ubicación para el dorso y el opérculo en alevinos de cachama blanca (Piaractus brachypomus). de la misma con una escala de 0 a 5 , donde 0 es nula, 3 es buena y 5 excelente, la encuesta se realizó a 10 personas ajenas al experimento, las cuales calificaron de acuerdo a su criterio y percepción.

\section{Análisis estadístico}

Para el análisis de los datos obtenidos durante el desarrollo del estudio; se utilizó el paquete estadístico SAS 9.1 (SAS Institute 2004). En todos los casos las diferencias se consideraron significativas si $\mathrm{P}<0,05$ y no significativas cuando $p>0,05$; el sesgo estandarizado y la curtosis estandarizada fueron usados para comprobar la normalidad de los datos tanto para peso como para longitud total; se realizó una comparación de medias mediante una prueba t-Student asumiendo varianzas iguales. Para evaluar la tasa de retención y la visibilidad en los experimentos estudiados se realizaron tablas de contingencia y pruebas de Chi-cuadrado de Pearson.

\section{Resultados}

\section{Parámetros de calidad de agua}

La temperatura promedio fue de, $25.71 \pm 0.88{ }^{\circ} \mathrm{C}$ durante el día, los demás parámetros evaluados se mantuvieron constantes durante todo el estudio. El $\mathrm{pH}$ estuvo en 6.2, la alcalinidad en $34.2 \mathrm{mg} / \mathrm{l}$, la dureza en $17.1 \mathrm{mg} / \mathrm{l}$ y la concentración de oxígeno disuelto en $8.0 \mathrm{mg} / \mathrm{l}$.

Los datos obtenidos muestran que el estudio estuvo dentro de las condiciones adecuadas para cultivo de peces dulceacuícolas (Merino, 2001), y según parámetros reportados por Poleo et al., (2011) en un cultivo de cachama blanca sometidas a altas densidades y en dos sistemas cerrados de recirculación de agua, comprendiendo; Temperatura de $26 \pm 1.2^{\circ} \mathrm{C}, \mathrm{pH} 7,3 \pm 0,2$, dureza $40-80 \mathrm{mg} / \mathrm{L}$ de $\mathrm{CaCO} 3$, y oxígeno disuelto $>4,7$ $\mathrm{mg} / \mathrm{L}$. Las condiciones de laboratorio de este estudio permitieron un mejor control de las características del agua y del estado sanitario de los animales reflejado en la sobrevivencia del $100 \%$ de los animales.

\section{Crecimiento}

Para peso corporal y talla, la tabla 1 describe estas variables de crecimiento a los 30 días de evaluación. Mediante el sesgo estandarizado y la curtosis estandarizada se comprobó que las muestras provenían de distribuciones normales tanto para peso como para longitud total. La Prueba t para comparar medias suponiendo varianzas iguales mediante el valor-P $>0,05$ demuestra que no existe diferencia estadísticamente 
significativa entre los pesos y la longitud total del grupo control y el VIE con un $95 \%$ de confianza. El tiempo promedio general de manejo por pez fue de 42 segundos.

Para ninguno de los experimentos, se encontró diferencias significativas entre los animales marcados y los no marcados con relación al crecimiento ( $\mathrm{P}>0.05)$; en el experimento 1 el promedio de peso de los individuos marcados y no marcados fue similar al inicio $(4,59 \pm 0.84$ y $4.52 \pm 0.93$ g respectivamente) y al final ( $16 \pm 3.6$ y $17 \pm 4.5$ g respectivamente); de igual manera; en el experimento 2, el promedio de peso de los individuos marcados y no marcados fue similar al inicio $(8,26 \pm 1,94$ y $8,58 \pm 2.02$ g respectivamente $)$ $y$ al final $(29.7 \pm 6,3$ y $28 \pm 7,2 \mathrm{~g})$. El coeficiente de variación tanto para peso como para talla indicó que los animales presentaron una alta homogeneidad para estas variables. Tal como se esperaba, el análisis de varianza explicó de una manera favorable el modelo para ambas variables $\left(R^{2}>0.600\right)$.

La sobrevivencia en ambos experimentos fue del $100 \%$; la tasa de crecimiento específica para los experimentos 1 y 2 fue similar tanto para los animales marcados como para los no marcados. En el experimento 1 los animales marcados y no marcados obtuvieron una tasa de crecimiento específica de $4.0 \% /$ día y $4.1 \% /$ día, igualmente en el experimento 2 los animales marcados y no marcados obtuvieron una tasa de crecimiento específica de $4.4 \%$ /día y $4.2 \%$ / día, respectivamente.

\section{Retención del implante VIE}

La retención en las dos posiciones corporales, dorso y opérculo en promedio fue de $94.1 \%$ y $57.5 \%$, respectivamente, para los dos experimentos, evidenciando que hubo diferencia entre las dos posiciones corporales como se puede evidenciar en la tabla 2; de particular interés el estadístico Chi-cuadrado que se usó para comparar la independencia entre los experimentos y la retención permitió aceptar la hipótesis nula y probar que el peso de los animales es independiente de la retención del marcaje, lo que quiere decir que las dos posiciones corporales del implante no presentaron diferencias significativas al compararlas así mismas en los dos experimentos.

La retención para el experimento 1 en el dorso y el opérculo fue de $94.7 \%$ y $55.2 \%$ respectivamente, para el caso del experimento 2 , fue de $93.4 \%$ y $59.7 \%$ respectivamente.

\section{Visibilidad}

La visibilidad con luz UV (ultravioleta) y sin luz UV en el opérculo fue de $96.6 \%$ y $77.7 \%$ y para el dorso fue de $92.6 \%$ y $61.5 \%$, respectivamente. De interés el estadístico Chi-cuadrado que se usó para comparar la independencia entre las dos posiciones de marcaje y el uso de luz UV y luz natural permitió aceptar la hipótesis nula y probar que la posición de marcaje es independiente de la visibilidad mediante luz UV y luz natural. Sin embargo, se halló discrepancia con relación a los colores fluorescentes y los no fluorescentes mediante luz natural y luz UV, demostrando preferiblemente el uso de los colores fluorescentes bajo luz UV.

\section{Discusión}

Los sistemas de marcaje internos se han descrito ampliamente en numerables especies, destacando la importancia de evaluar la utilidad real y aprovechamiento que tiene cada especie (Soula et al., 2009).

Tabla 1. Peso corporal y talla (longitud total) (media \pm desviación estándar) del experimento 1 y 2 a los 30 días de evaluación para cachama blanca (Piaractus brachypomus).

\begin{tabular}{|c|c|c|c|c|c|c|c|c|}
\hline \multirow{2}{*}{ Experimento } & \multicolumn{3}{|c|}{ Peso Corporal (g) } & \multicolumn{3}{c|}{ Talla (mm) } \\
\cline { 2 - 9 } & \multicolumn{2}{|c|}{ Control } & \multicolumn{2}{|c|}{ VIE } & \multicolumn{2}{c|}{ Control } & \multicolumn{2}{c|}{ VIE } \\
\cline { 2 - 9 } & & CV $\%$ & & CV \% & & CV \% & \multicolumn{2}{c|}{ CV \% } \\
\hline \multirow{2}{*}{ E 1 } & $16^{\mathrm{a}} \pm 3,6$ & 22,3 & $17^{\mathrm{a}} \pm 4,5$ & 26,5 & $91,8^{\mathrm{a}} \pm 7,4$ & 8,12 & $92,8^{\mathrm{a}} \pm 8,2$ & 8,8 \\
\hline \multirow{2}{*}{ E 2 } & $29,7^{\mathrm{a}} \pm 6,3$ & 21 & $28^{\mathrm{a}} \pm 7,2$ & 25,8 & $112,3^{\mathrm{a}} \pm 7,6$ & 6,8 & $109,8^{\mathrm{a}} \pm 10$ & 9,2 \\
\hline
\end{tabular}

Letras diferentes entre tratamientos denotan diferencias significativas $(P<0.05)$. Coeficiente de variación $(C V)$ 
Tabla 2. Tasa de retención en dorso y opérculo para los experimentos 1 y 2 a los 30 días de evaluación para cachama blanca (Piaractus brachypomus).

\begin{tabular}{|c|c|c|}
\hline Retención (\%) & Experimento 1 & Experimento 2 \\
\hline Dorso & 94,7 & 93,4 \\
\hline Opérculo & 55,2 & 59,7 \\
\hline
\end{tabular}

Las diferencias anatómicas, las diferentes condiciones de calidad de agua, las diversas magnitudes de resistencia, la susceptibilidad a la manipulación y términos de crecimiento, son unas de las razones que fundamentan su evaluación para cada especie (Davis et al., 2004, Jerry et al., 2001). En este sentido, para este estudio la ausencia de diferencias en el peso, talla y tasa de crecimiento específica entre peces marcados y no marcados en ambos experimentos, concuerda con lo reportado por Soula et al., (2012) en el bocinegro (Pagrus pagrus), quien evaluó el VIE en relación con el crecimiento, mortalidad, tiempo de manejo y tasa de perdida, encontrando que no existió efecto alguno sobre las variables, al igual que lo demostrado por otros autores (Soula et al., 2009, Zeeh y Wood, 2009, Liu et al., 2011). De igual manera revela que no hay efecto sobre el crecimiento de los peces de 4.59 y 8.9 g. Estos pesos en promedio demuestran que son los más adecuados para iniciar el marcaje con VIE, según estudios similares que han examinado su efecto en otras especies de peces, por ejemplo, el nulo efecto sobre el crecimiento fue demostrado en peces de dorada (Sparus auratus L) de 7 a 18 gramos (Astorga et al., 2005), en peces de bocinegro de 2 gramos, de igual manera como lo mencionó Jerry et al., (2001). Con referencia a la sobrevivencia del $100 \%$ obtenida en este estudio se demuestra por una parte, que no existe efecto alguno del VIE que se relacione con la salud de los especímenes como lo describe Soula et al. (2009) para animales superiores a 2 gramos, por otra parte, la alta capacidad de resistencia al manejo de alevinos de cachama blan$\mathrm{ca}$, que a pesar de ser el máximo tiempo reportado por animal (42 segundos), frente a lo conseguido en dorada por Astorga et al., (2005) de 15.7 segundos con $4 \%$ de mortalidad y lo reportado por Bailey et al., (1998) en coho salmon (Oncorhynchus kisutch) que solo necesitó de 12 segundos por pez con un 5\% de mortalidad a las 24 horas después de la marcación; estos autores concluyen que el tiempo de manejo en general depende de la destreza del implantador.

En relación con la retención del implante, se han referenciado diferentes estimados como por ejemplo Soula et al., (2012), quienes obtuvieron $75 \%$ de reten- ción en bocinegro durante 15 y 30 días de estudio en dos tallas diferentes. Para este estudio de 30 días, un $94 \%$ en promedio para la posición corporal del dorso demuestra una elevada confiabilidad en su uso, por encima del $57.5 \%$ obtenido en el opérculo, reconociendo que aún falta evaluar esta variable por mucho más tiempo.

A manera de colofón, el estudio pone de manifiesto que es factible el uso del V.I.E. en animales de peso de 4,5 g en adelante y que la posición más viable para su uso es el dorso. Preferiblemente se debe utilizar los colores fluorescentes bajo exposición a luz UV. Finalmente el uso de este sistema de identificación, permitirá proyectar programas de cría selectiva y mejoramiento genético en la especie, una vez se estime el efecto de la genética sobre características de interés económico.

\section{Agradecimientos}

Los autores agradecen a la Dirección General de Investigaciones (DGI) de la Universidad de los Llanos por el apoyo financiero (proyecto código CAIALL-2-2013), así como al programa de Jóvenes Investigadores de Colciencias por la manutención de los jóvenes suscritos Felipe Bernal y Jeferson Valderrama.

\section{Bibliografía}

Astorga N, Afonso JM, Zamorano MJ, Montero D, Oliva V, Fernán$\operatorname{dez} \mathrm{H}$, Izquierdo MS. Evaluation of visible implant elastomer tags for tagging juvenile gilthead seabream (Sparus auratus L.); effects on growth, mortality, handling time and tag loss. Aquacult Res. 2005;36(8): 733-738.

Bailey R, Irvine J, Dalziel F, Nelson T. Evaluations of visible implant fluorescent tags for marking coho salmon smolts. N Am J Fish Manage. 1998;18(1):191-196.

Baras E, Malbrouck C, Houbart M, Kestemont P, Mélard C. The effect of PIT tags on growth and physiology of age-0 cultured Eurasian perch Perca fluviatilis of variable size. Aquaculture. 2000;185(1-2): 159-173.

Baras E, Westerloppe L, Mélard C, Philippart J-C, Benech V. Evaluation of implantation procedures for PIT-tagging juvenile Nile tilapia. N Am J Aquacult. 1999;61(3): 246-251.

Bernal F, Gallego F. Estimación de parámetros genéticos para peso y talla a diferentes edades en yamú (Brycon amazonicus). Revista UDCA Actualidad \& Divulgación Científica. 2016;19(1):123130.

Brennan NP, Leber KM, Blackburn BR. Use of coded-wire and visible implant elastomer tags for marine stock enhancement with juvenile red snapper Lutjanus campechanus. Fish Res. 2007;83(1):90-97. 
Davis JLD, Young-Williams AC, Hines AH, Zmora O. Comparing two types of internal tags in juvenile blue crabs. Fish Res. 2004;67(3): 265-274.

FAO - Organización de las Naciones Unidas para la Alimentacion y la Agricultura. 2014. El Estado Mundial de la Pesca y la Acuicultura. Roma, Italia.

Jerry DR, Stewart T, Purvis IW, Piper LR. Evaluation of visual implant elastomer and alphanumeric internal tags as a method to identify juveniles of the freshwater crayfish, Cherax destructor. Aquaculture. 2001;193(1-2):149-154.

Leiva AFA, BONILLA DJP, MOJICA AS, MURCIA CT. 2007. PESCA Y ACUICULTURA COLOMBIA 2007. Ministro de Agricultura y Desarrollo Rural.

Liu Z-M, Wang G-Z, Ye H-H, Li S-J, Tao Y, Lin Q-W, Mohammed EH. Tag performance and physiological responses of juvenile mud crabs Scylla paramamosain tagged with visible implant elastomer. Fish Res. 2011;110(1):183-188.

Mesa-Granda M, Botero-Aguirre M. La cachama blanca (Piaractus brachypomus), una especie potencial para el mejoramiento genético. Rev Colomb Cienc Pec. 2007;20(1):79-86.

Mesa-Granda M, Cerón-Muñoz M, Olivera M, Botero-Aguirre M. 2006. RAYOS X: UNA HERRAMIENTA PARA LA CUANTIFICACIÓN DE ALGUNAS ESTRUCTURAS ÓSEAS EN CACHAMA BLANCA, PIARACTUS BRACHYPOMUS (CUVIER, 1818).
Nascimento AF, Maria AN, Pessoa NO, Carvalho MAM, Viveiros ATM. Out-of-season sperm cryopreserved in different media of the Amazonian freshwater fish pirapitinga (Piaractus brachypomus). Anim Reprod Sci. 2010;118(2-4): 324-329.

Poleo G, Aranbarrio JV, Mendoza L, Romero O. Cultivo de cachama blanca en altas densidades y en dos sistemas cerrados. Pesq Agropec Bras, Brasília. 2011;46(4):429-437.

Sastre OF, Hernández G, Cruz Casallas P. Influencia del peso corporal y de la temperatura del agua sobre el consumo de oxígeno de la Cachama Blanca (Piaractus brachypomus). Rev Colomb Cienc Pec. 2009;17(4): 11-16.

Soula M, Navarro A, Hildebrandt S, Zamorano MJ, Roo J, Hernández-Cruz CM, Afonso JM. Evaluation of VIE (Visible Implant Elastomer) and PIT (Passive Integrated Transponder) physical tagging systems for the identification of red porgy fingerlings (Pagrus pagrus). Aquac Int. 2012;20(3): 571-583.

Soula M, Navarro A, Zamorano MJ, Roo J, Real F, Ginés R, et al. Evaluación de los sistemas Visible Implant Elastomer (VIE) y Passive Integrated Transponder (PIT) para marcar alevines de bocinegro (Pagrus pagrus): efectos sobre el crecimiento, la mortalidad y la tasa de retención. ITEA. 2006;102(2):159-167

Zeeh KM, Wood JB. Impact of visible implant elastomer tags on the growth rate of captive Caribbean reef squid Sepioteuthis sepioidea. Fish Res. 2009;95(2-3):362-364. 